\title{
The Diagnostic Accuracy of Purple Line in Prediction of Labor Progress in Omolbanin Hospital, Iran
}

\author{
Masoumeh Kordi ${ }^{1}$; Morvarid Irani ${ }^{1,} ;$ Fatemeh Tara $^{1} ;$ Habibollah Esmaily $^{2}$ \\ ${ }^{1}$ Department of Midwifery, School of Nursing and Midwifery, Mashhad University of Medical Sciences, Mashhad, IR Iran \\ ${ }^{2}$ Departement of Biostatistics and Epidemiology, School of Health, Mashhad University of Medical Sciences, Mashhad, IR Iran \\ ${ }^{*}$ Corresponding Author: Morvarid Irani, Department of Midwifery, School of Nursing and Midwifery, Mashhad University of Medical Sciences, Mashhad, IR Iran. Tel: +98-5117531407, \\ E-mail: Irani.morvarid@gmail.com
}

Received: November 20, 2013; Revised: September 5, 2014; Accepted: September 23, 2014

\begin{abstract}
Background: Currently, vaginal examination is the gold standard for assessment of labor progress. The World Health Organization emphasizes that the number of vaginal examinations should be limited where it is necessary.

Objectives:Therefore, this study aimed to determine the diagnostic accuracy of purple line in the prediction of labor progress.

Patients and Methods: In this cross-sectional study, 350 women with a single pregnancy in vertex presentation and gestational age of 38-42 weeks without any medical disorder, admitted to government hospitals of Mashhad, were selected using convenience sampling. Vaginal examination and observation of the line each hour in the active phase of labor were measured. Abnormal progress of labor was defined as cervical dilatation less than 1 centimeter/hour in the active phase for two consecutive hours and fetal head descend less than $1 \mathrm{~cm} / \mathrm{h}$ or duration of more than two hours for nulliparous and one hour for multiparous In the second stage of labor. Data was analyzed by SPSS version 16 using chi -square test.

Results: The purple line appeared in $75.3 \%$ of women during the active phase of labor. Appearance of the purple line in the prediction of labor progress had $90.2 \%$ sensitivity, $45.3 \%$ specificity, $88.1 \%$ positive predictive value, $51.0 \%$ negative predictive value in the first stage of labor and had $87.6 \%$ sensitivity, $52.4 \%$ specificity, $96.5 \%$ positive predictive value, $22.0 \%$ negative predictive value in the second stage of labor and has $68.57 \%$ sensitivity, $42.66 \%$ specificity, $85.32 \%$ positive predictive value, and $43.85 \%$ negative predictive value for the total labor.

Conclusions: According to the appearance of the purple line in most of the cases and its high sensitivity and specificity, we can use it as a non-invasive complementary method for clinical assessment of labor progress.
\end{abstract}

Keywords:First Stage Labor; Second Stage Labor; Vaginal examinations; Sensitivity and Specificity; Cervical Dilatation

\section{Background}

One of the key aspects of maternal care is observing labor progress; and lack of labor progress is one of a couple of causes for converting to cesarean operations. Therefore, focusing on the labor progress is very important in the decision to intervene in normal vaginal delivery $(1,2)$. There are several methods for assessing labor progress including: uterine contractions, descent and fetal head position by abdominal palpation, vaginal examination to determine cervical dilatation and observing changes in women behavior and appearance $(2,3)$ Currently, the standard method for assessing progress of labor, particularly in developing countries, is Partograph that is used for detecting normal and abnormal progress of labor. World Health Organization in 2003 has provided a compilation Partograph for managing vaginal delivery. They demonstrated that cervical dilatation of less than one centimeter per hour for two consecutive hours is an indicator of abnormal progress of labor (3). Currently, vaginal examination is the gold standard for assessment of labor progress, which in addition to cervical dilatation and descent, is used to determine the fetal head position, presentation of the fetus, length and size of the cervix, pelvic capacity, fetal head molding and the proportion of fetuses with the pelvic bones (2, 4). However, most vaginal examinations are done only to assess the progress of cervical dilatation (3). Some studies have shown that the overall accuracy for cervical dilatation is between $48 \%$ to $56 \%$ with $\pm 1 \mathrm{~cm}$ error, to $89.5 \%$ to $91.7 \%$ and this accuracy is reduced with the increase of cervical dilatation $(5,6)$. Huhn et al. noted that soft cervical models of cervical dilatation measurements were only $19 \%$ accurate and hard cervical models were $54 \%$ (7) accurate. Buchmann study showed that there is a difference between two midwives in $11 \%$ of cases in $\geq$ $2 \mathrm{~cm}$ of cervical dilatation measurements (6). Considering the fact that assessment of labor progress is based on measurements of cervical dilatation, there is concern about unnecessary interventions such as amniotomy, oxytocin use and cesarean section that are based on this criteria (8-10). Therefore, to reduce such unnecessary interventions, labor progress based on other alternative or complementary measures of cervical dilation is neces-

Copyright (C) 2014, Iranian Red Crescent Medical Journal. This is an open-access article distributed under the terms of the Creative Commons Attribution-NonCommercial 4.0 International License (http://creativecommons.org/licenses/by-nc/4.0/) which permits copy and redistribute the material just in noncommercial usages, provided the original work is properly cited. 
Kordi M et al.

sary and less invasive methods of assessing progress in labor should be investigated. Vaginal examinations can be unpleasant, intrusive and embarrassing for women $(11,12)$. Indiscriminate use, login to the privacy of women and repeated vaginal examinations can cause physical and psychological discomfort in women (13). On the other hand, there is a link between the numbers of vaginal examinations, the risk of puerperal sepsis, urinary tract infections, and early neonatal infections (14-16). The National Institute for Health and Clinical Excellence (NICE) recommend that vaginal examinations should not be routinely performed and should be done every four hours in the first stage of labor (17) .'Keeping Childbirth Natural and Dynamic' (KCND Scotland) has been developed in an attempt to limit interventions in normal labor and encourage a more holistic and less prescriptive approach to labor care. They tried to limit the number and frequency of vaginal examinations (18). In one study, the average number of vaginal examinations for the diagnosis of labor was three, ranged between 0 and 11 , in which the average duration of labor was 8 hours was variable (19). The WHO recommends that the number of vaginal examinations should be limited to those which are strictly necessary and, ideally, this should be the one examination to establish active labor (18). Walsh suggests that "repetition of routine vaginal examinations in normal labor should be abandoned until research establishes their appropriate place" (20). Over the last 10 years there has been an increased interest in reducing intervention in normal labor. As NICE recommend, further research are needed to assess the relation between the frequency of vaginal examination and risk associated with vaginal examinations (17). Considering the increasing worldwide concern about the use of routine intervention in labor, the need of an alternative and less intrusive method for assessing progress in labor is totally sensed. Prior to the 1970s, midwives had more emphasis on alternative methods of assessing labor progress (21). It seems necessary in order to minimize unnecessary interventions in labor, using non-invasive methods (22). Some studies have described behaviors and vocalizations indicative of the progress of labor $(17,23,24)$. But these methods whether as alternative or complementary to vaginal examinations have the least reliability. One of the non-invasive methods to assess the progress of cervical dilatation and fetal head descent in labor is the purple line. Hobbs is the first Person who mentioned purple line, which appeared as a purple point around anus during labor and progresses along with cervical dilation. Direction of this progress is upward from intergluteal line to sacro-coccygeal joint like a thermometer in which mercury column moves upward (24) (Figure 1).

Byrne and Edmonds were the first to document the appearance of a line of red/purple discoloration seen to arise from the anal margin and extend between the buttocks reaching the nape of the buttocks at the onset of the active stage of labor. They conducted a study with 48 women in spontaneous labor and noted that the purple line was seen on $89 \%$ of the labors. Significant correlation was seen between the length of the purple line and cervical dilatation and the station of the fetal head (25). Researchers suggested that this congestion possibly occurs because of intrapelvic pressure as the fetal head descends, which may account for the correlation between station of the fetal head and purple line length. The rhombus of Michaelis is a kite shaped area over the lower back that includes the lower lumbar vertebrae and sacrum. It is believed that this area of bone moves backwards during advanced labor, pushing out the wings of the ilea and increases the pelvic diameter $(21,25)$. Shepherd et al. in Scotland noted that the purple line was seen on $76 \%$ of occasions. Significant correlation was detected between the length of the purple line and cervical dilatation and the station of the fetal head. The purple line was significantly

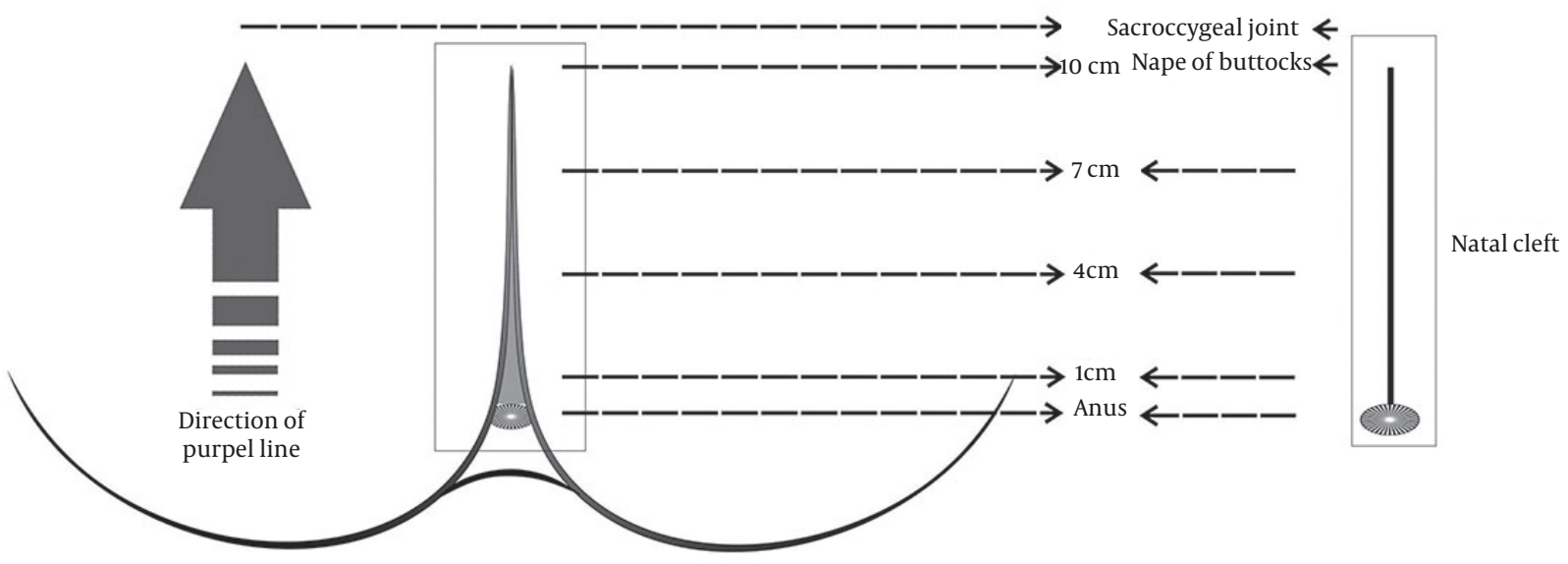

Figure 1. Direction of Purple Line 
Kordi M et al.

more likely to be present in women in spontaneous labor, when compared with those women admitted for induction of labor. They suggested that more research is needed for clinical application in the assessment of labor progress (21). Narchi et al. in Brazil noted that the purple line was seen on $56 \%$ of the occasions and similarly, there was a significant correlation between the length of the purple line and cervical dilatation (12). We can identify the clinical application of this method by determining the diagnostic accuracy of purple line in the prediction of labor progress as a non-invasive method. It could be used as an alternative or supplement to the vaginal examination, if it shows a high diagnostic accuracy for predicting the progress of labor. Thus, we can reduce the number of vaginal examinations and subsequently the risk of maternal and neonatal infections.

\section{Objectives}

The objective of our study was to determine the percentage of appearance of purple line during the labor and its diagnostic accuracy to predict labor progress in the first and second stage of labor in the nulliparous and multiparous women, who referred to Ommolbanin Hospital of Mashhad between April and August 2012.

\section{Patients and Methods}

In this cross-sectional study, 350 nulliparous and multiparous women, who had enrolled in maternity ward of Omolbanin Hospital (a tertiary specialized hospital in Mashhad, northeast of Iran with approximately 8,200 births per year), were studied. They enrolled in the study since April to August 2012. A total of 370 women were selected from people who attended clinics for vaginal delivery and had the inclusion criteria using non-probability sampling method of which 356 were accepted to participate in the study and 350 were followed up until delivery. The sample size was determined after doing the pilot study on 50 occasions with 95\% confidence interval. Before the study, the coordinator explained details of the research protocol and gave them an ample amount of time to ask questions from the coordinator who were involved in the trial to the potential participants. Subsequently, eligible parturient signed the informed consent. The principle of the study protocol was approved by the Ethic Committee of Mashhad University of Medical Sciences (Approval No. 900759 in April 2012). Women who had single pregnancy in vertex presentation, gestational age of 38-42 weeks without medical disorder entered the study. At 3-4 cm cervical dilatation the researcher observed and recorded the presence or absence of purple lines between the buttocks in the lateral position every hour in the active phase of the labor until the delivery was performed. The presence of purple line was discoloration (red/purple) between the buttocks. Then vaginal examination (cervical dilation and station of fetal head) was performed by the help of the researcher who was out of the room and did not know of the consequences or presence or absence of the purple line.as the gold standard The results of these measurements were recorded in Partograph and delivery information form. These measurements were repeated every 1 hour in the active phase of the labor until the delivery was performed. Abnormal progress of labor was defined as cervical dilatation less than 1 centimeter/hour in the active phase for two consecutive hours and fetal head descend less than $1 \mathrm{~cm} / \mathrm{h}$ or duration of more two hours for nulliparous and one hour for multiparous women in the second stage of labor.

Equipment used in this study include: Demographic characteristics checklist, Partograph and delivery progress information record form and purple line observation record form. The validity of demographic characteristics checklist, Partograph and delivery progress information record form by content validity. In this way, after literature review, demographic characteristics checklist, Partograph and delivery progress information record form prepared, then validity of them determined by 10 member of the faculty and content validity index was calculated $(\mathrm{CVI}=90 \%)$. The researcher's skills for observing purple line and the help of the researcher's skills for vaginal examination were confirmed by five training sessions at the presence of the supervisor and specialist consultant. The reliability of demographic characteristics checklist, was determined by test-retest. The reliability Partograph and delivery progress information record form, was determined by Interobserver reliability and the resultant correlation was confirmed $(\mathrm{r}=0.845)$. The reliability of the researcher's skills for observation purple line was determined by Interobserver reliability and the resultant correlation was confirmed $(r=0.85)$. After delivery, the subjects were divided into normal and abnormal labor progress, and diagnostic accuracy was calculated based on the presence or absence of purple lines. Data was analyzed by SPSS version 16 using Kolmogorov-Smirnov and chi-square test. Sensitivity, specificity, positive and negative indicative values were calculated manually.

\section{Results}

Three hundred and seventy women were invited to participate of which, 356 (96\%) completed the study. Of those who agreed to participate, the data collection commenced but was incomplete for two women and the data was missing for four women. Complete data for cervical dilatation and length of line was recorded throughout labor for 350 women. For realizing normality in data we used K-S test. The mean (SD) age of the women in this study was $25.22( \pm 5)$ years. The mean (SD) gestation at birth was 39.8 ( \pm 1.2 ) weeks and the mean (SD) birth weight were 3332 ( \pm 1543 ) grams. Of the 350 women who enrolled, there were $61.2 \%$ primigravidas and $38.8 \%$ multigravidas. The demographic and clinical characteristics are shown in Table 1. 
Table 1. Demographic and Clinical Characteristic of the Study Population $(\mathrm{n}=350)^{\mathrm{a}}$

\begin{tabular}{lc}
\hline Variable & Values \\
\hline Age, $\mathbf{y}$ & $25.22 \pm 5$ \\
\hline Gestation at birth, weeks & $39.8 \pm 1.2$ \\
\hline Birth Weight, gr & $3332 \pm 1543$ \\
\hline BMI, kg/m & $24 \pm 5.2$ \\
\hline Illiterate & 0 \\
\hline Primary & $117(33.5)$ \\
\hline Intermediate & $204(58)$ \\
\hline University & $29(8.5)$ \\
\hline Primiparous & $222(63.4)$ \\
\hline Multiparous & $128(36.6)$ \\
\hline Spontaneous Labor & $305(87.1)$ \\
\hline Induction & $45(22.9)$ \\
\hline Vaginal Delivery & $329(94)$ \\
\hline Forceps-vacuum & $5(1.4)$ \\
\hline C.Section & $16(4.6)$ \\
\hline
\end{tabular}

${ }^{\mathrm{a}}$ Data are presented as Mean \pm SD or No. (\%).
Furthermore, 78.6\% were in normal labor progress ( $\mathrm{n}=$ 275 ) and $21.4 \%$ were in abnormal labor progress $(n=75)$. The total number of VEs carried out was 1581 occasions. The line was present for $75.3 \%$ of the occasions. The percentage of examinations where a purple line was present increased with an increase in cervical dilatation from $66.4 \%$ with a cervical dilatation of $3 \mathrm{~cm}$ to $84 \%$ when cervical dilatation was $10 \mathrm{~cm}$. In $90.2 \%$ of the subjects with normal labor progress of the first stage of labor, there was a purple line, present in the $54.7 \%$ of the subjects with abnormal labor progress. In $87.6 \%$ of the subjects with normal labor progress of the second stage of labor, there was a purple line, present in the $47.6 \%$ of the subjects with abnormal labor progress. In $90.9 \%$ of the subjects with normal labor progress of total stage of labor, there was a purple line, present in the $57.3 \%$ of subjects with abnormal labor progress. Chi-square test results showed that there is a significant association between the presence and absence of purple line with labor progress (Table 2).

Appearance of the purple line in the prediction of labor progress has $90.2 \%$ sensitivity, $45.3 \%$ specificity, $88.1 \%$ positive predictive value, $51.0 \%$ negative predictive value in the first stage of labor, $87.6 \%$ sensitivity, $52.4 \%$ specificity, $96.5 \%$ positive predictive value, $22.0 \%$ negative predictive value in the second stage of labor and $68.57 \%$ sensitivity, $42.66 \%$ specificity, $85.32 \%$ positive predictive value, and 43.85\% negative predictive value for total labor (Table 3 ).

\begin{tabular}{|c|c|c|c|c|}
\hline \multirow[t]{2}{*}{ Existence of Purple Lines } & \multicolumn{3}{|c|}{ labor Progress } & \multirow[t]{2}{*}{ Chi-Square Test } \\
\hline & Normal Labor progress & $\begin{array}{c}\text { Abnormal Labor } \\
\text { Progress }\end{array}$ & $\begin{array}{l}\text { Total Labor } \\
\text { Progress }\end{array}$ & \\
\hline First stage of labor & & & & $\mathrm{Df}=1, \mathrm{P}<0.001$ \\
\hline Yes & $258(90.2)$ & $35(54.7)$ & $293(83.7)$ & \\
\hline No & $28(9.8)$ & $29(45.3)$ & $57(16.3)$ & \\
\hline Total & $286(100.0)$ & $64(100.0)$ & $350(100.0)$ & \\
\hline Second stage of labor & & & & $\mathrm{df}=1, \mathrm{P}<0.001$ \\
\hline Yes & $275(87.6)$ & $10(47.6)$ & $285(85.1)$ & \\
\hline No & $39(12.4)$ & $11(52.4)$ & $50(14.9)$ & \\
\hline Total & $314(100.0)$ & $21(100.0)$ & $335(100.0)$ & \\
\hline Total stage of labor & & & & $\mathrm{df}=1, \mathrm{P}<0.001$ \\
\hline Yes & $250(90.9)$ & $43(57.3)$ & $293(83.71)$ & \\
\hline No & $25(9.1)$ & $32(42.7)$ & $57(16.29)$ & \\
\hline Total & $275(100.0)$ & $75(100.0)$ & $350(100.0)$ & \\
\hline
\end{tabular}

Table 3. Diagnostic Value of Presence or Absence of Purple Lines in Predicting Labor Progress in First, second and Total Stage ${ }^{a}$

\begin{tabular}{lcccccc}
\hline $\begin{array}{l}\text { Presence or Absence of } \\
\text { Purple Lines }\end{array}$ & Sensitivity & Specificity & $\begin{array}{c}\text { Positive Indicative } \\
\text { Value }\end{array}$ & $\begin{array}{c}\text { Negative Indicative } \\
\text { Value }\end{array}$ & Accuracy & pLR \\
\hline First stage of labor, No. & 90.2 & 45.3 & 88.1 & 51.0 & 82.0 & 2.03 \\
Second stage of labor, No. & 87.6 & 52.4 & 96.5 & 22.0 & 85.4 & 1.7 \\
Total labor, No. & 68.75 & 42.66 & 85.32 & 43.85 & 80.57 & 1.9 \\
\hline
\end{tabular}

\footnotetext{
${ }^{\mathrm{a}}$ PLR, Positive Likelihood Ratio.
} 
Kordi M et al.

\section{Discussion}

In our study, the purple line appeared in $75.3 \%$ of the women during the active phase of labor. The presence of purple line increased with an increase in cervical dilatation. This number increase from $66.4 \%$ with a cervical dilatation of $3 \mathrm{~cm}$ to $84.0 \%$ when cervical dilatation was $10 \mathrm{~cm}$. In Bryne and Edmonds the purple line appeared in $89 \%$ of the women (25). These differences could be because they had a different sample size or methods. They conducted a small study with 48 women in spontaneous labor; but we conducted a study with 350 women in spontaneous and induction labor. In their study, all the line and vaginal observations and examinations were made by the same midwife, but in our study, all examinations of the line were checked by two midwives for increasing the confidence in the findings. Women in their study were examined by different midwives, which reduced the accuracy of vaginal examination. In Shepherd et al. study, the purple line appeared in $76 \%$ of the women and the results of our study are in agreement with their results (21). In Narchi et al. study, the purple line appeared in 56\% of the women. There was a significant association between white women and nonwhite women; purple line was present in $67.5 \%$ of the whites and $48.5 \%$ in the nonwhite women (12). In our study, the appearance for the purple line was higher than that in the above-mentioned studies, which would be due to different sample size or different race and skin types. The appearance for the purple line in nonwhite women is less than white women. Bryne and Edmonds suggested that this congestion possibly occurs because of intrapelvic pressure as the fetal head descends which may account for the correlation between station of the fetal head and purple line length. The rhombus of Michaelis is a kite shaped area over the lower back that includes the lower lumbar vertebrae and sacrum. It is believed that this area of bone moves backwards during advanced labor, pushing out the wings of the ilea and increases the pelvic diameter (25). In our study, from 350 women, 78.6\% had normal labor progress $(n=275)$ and $21.4 \%$ abnormal labor progress $(n=75)$. In the first stage of labor $81.7 \%$ had normal labor progress and $18.7 \%$ abnormal labor progress and in the second stage of labor 89.7\% had normal labor progress and $6.0 \%$ abnormal labor progress. In another study, there was $87.5 \%$ normal labor progress and $17.25 \%$ abnormal labor progress. These differences could be because they had different definitions of labor progress. They defined abnormal labor progress as cesarean section or vacuum extraction, while in the presence of effective uterine contractions in active phase of labor, the rate of cervical dilation was less than $1 \mathrm{~cm}$ per hour for two hours, and the rate of dilation was less than $1 \mathrm{~cm}$ per hour in the second phase of delivery. In addition, in their study, only primiparous women were enrolled, whereas in our study, primiparous and multiparous women were enrolled (26).

Our results showed that the normal progression of la- bor was higher in the group for which the purple line appeared.Appearance of the purple line in the prediction of labor progress in the first stage of labor has $90.2 \%$ sensitivity, $45.3 \%$ specificity, $88.1 \%$ positive predictive value, and $51.0 \%$ negative predictive value and in the second stage of labor it has $87.6 \%$ sensitivity, $52.4 \%$ specificity, $96.5 \%$ positive predictive value, negative $22.0 \%$ predictive value and the total labor has $68.57 \%$ sensitivity, $42.66 \%$ specificity, $85.32 \%$ positive predictive value, and $43.85 \%$ negative predictive value. The purple line appearing with relatively high sensitivity and specificity is capable to predict the progress of labor in the first, second and total stage of labor, whereas other methods of assessing the labor progress can only identify at same moment. Actually, in the first stage of labor by observing the purple line, which is a very easy and non-invasive method compared with the gold standard can predict the labor progress. Vaginal examination for assessment of the cervix dilation is currently considered to be the gold standard for assessment of labor progress but it is an invasive method and has some complication for women including an increase maternal and neonatal infections, painful and unpalatable. Pornprasertsuk et al. conducted a study to determine the diagnostic value of alteration of sacral pain and labor progression in latent phase of labor. The results from this study showed that the increase in sacral pain tended to have an increase in cervical progression, which was defined as the increase in cervical effacement by at least $20 \%$ and/or increase in cervical dilatation in discrete number at least $1 \mathrm{~cm}$ with $92.75 \%$ sensitivity, $85.71 \%$ specificity, $98.46 \%$ positive predictive value and $54.55 \%$ negative predictive value, respectively (26). These differences could be because they assessed labor progress in the latent phase of the first stage of labor; but we assessed labor progress in the active phase of the first stage. On the other hand, they just measured pain at hour 0 and two, while our measurements were performed in active phase of labor per hour until childbirth. Also, purple line criterion was measured in our study and sacral pain criterion was measured in their study.

In this study, we are aware that methodologically, the measurements of labor progress (cervical dilatation and station of fetal head) and presence and absence of the purple line were blinded. We used only one midwife (researcher) to observers in total cases in order to minimize the confounding factors and another midwife for vaginal examination and determining labor progress (to help the researcher) who was out of the room and did not know of the consequences of presence or absence purple line. The limitations of this study are the measurements of cervical dilatation and descending of the fetus head, which are subjective and there is no direct accurate equipment to measure. Also, vaginal examination is considered as the gold standard. According to appearance of purple line in the most cases and its high sensitivity and 
specificity, we can use it as a non-invasive method for assessment of labor progress at clinics.

\section{Acknowledgements}

The study is a part of a research thesis approved by Mashhad University of Medical Sciences in April 2012, with the approval code of 900759 . It has been carried out through financial supports of its research deputy. Hereby, the deputy's assistance and cooperation to perform the study are highly appreciated.

\section{Authors' Contributions}

Masoumeh Kordi and Morvarid Irani developed the original idea and the protocol, abstracted and analyzed data, wrote the manuscript. Fatemeh Tara contributed to the development of the protocol, abstracted data, and prepared the manuscript. Statistical analysis was done by Habibollah Esmaily and Morvarid Irani.

\section{Funding/Support}

This study was supported in part by grant CA34988 from Mashhad University of Medical Sciences and by a teaching from the Mashhad University of Medical Sciences (Mrs kordi).

\section{References}

1. Benfield R. Williams Obstetrics, 21st Edition. J Midwifery Women Health. 2003;48(5):369.

2. Midwives TRCo.. Evidence Based Guidelines for Midwifery-Led Care in Labour: Assessing Progress in Labour Practice Points; 2012.

3. Duff M. . A study of labour.. Sydney: University of Technology; 2005

4. Shepherd A, Cheyne H. The frequency and reasons for vaginal examinations in labour. Women Birth. 2013;26(1):49-54.

5. Phelps JY, Higby K, Smyth MH, Ward JA, Arredondo F, Mayer AR. Accuracyand intraobserver variability of simulated cervical dilatation measurements. Am J Obstet Gynecol.1995;173(3 Pt1):942-5.

6. Buchmann EJ, Libhaber E. Accuracy of cervical assessment in the active phase of labour. BJOG. 2007;114(7):833-7.

7. Huhn KA, Brost BC. Accuracy of simulated cervical dilation and effacement measurements among practitioners. Am J Obstet Gynecol. 2004;191(5):1797-9.

8. Letic M. Inaccuracy in cervical dilatation assessment and the progress of labour monitoring. Med Hypotheses. 2003;60(2):199-201.
9. Mold JW, Stein HF. The cascade effect in the clinical care of patients. N Engl J Med.1986;314(8):512-4.

10. Tracy SK, Sullivan E, Wang YA, Black D, Tracy M. Birth outcomes associated with interventions in labour amongst low risk women: a population-based study. Women Birth. 2007;20(2):41-8.

11. Lewin D, Fearon B, Hemmings V, Johnson G. Women's experiences of vaginal examinations in labour. Midwifery. 2005;21(3):267-77.

12. Narchi NZ, Camargo DCSC, Salim NR, Menezes MDO, Bertolino MM. The use of the "purple line" as an auxiliary clinical method for evaluating the active phase of delivery. Rev Bras Saúde Matern Infant. 2011;11(3):313-22.

13. Menage J. Post-traumatic stress disorder following obstetric gynaecological procedures. Br J Midwifery. 1996;4(10):532-3.

14. Seaward PG, Hannah ME, Myhr TL, Farine D, Ohlsson A, Wang EE, et al. International multicenter term PROM study: evaluation of predictors of neonatal infection in infants born to patients with premature rupture of membranes at term. Premature Rupture of the Membranes. Am J Obstet Gynecol. 1998; 179(3 Pt 1):635-9

15. Maharaj D. Puerperal Pyrexia: a review. Part II. Obstet Gynecol Surv 2007;62(6):400-6.

16. Wong A, Rosh A. Pregnancy. Postpartum Infections. eMedicine. Available from: http://emedicine.medscape.com/article/796892overview.

17. National Institute for Health and Clinical Excellence.. Clinical Guideline 55 Intrapartum care. 2007. Available from: http://guidance.nice.org.uk/CG55/Guidance/pdf/English.

18. NHSWales.. All Wales Clinical Pathway for Normal Labourb. 2006. Available from: http://www.wales.nhs.uk/sites3/home cfm?orgid $=327$.

19. Scottish Government.. Keeping Childbirth Natural and Dynamic 2009. Available from: http://www.healthcareimprovementscotland.org/our_work/reproductive, maternal_child/programme_resources/http://www.healthcareimprovementscotland.org/our_work/reproductive.

20. Walsh D. Part three: Assessing womens progress in labour. $B r J$ Midwifery. 2000;8(7):449-57.

21. Shepherd A, Cheyne H, Kennedy S, McIntosh C, Styles M, Niven C. The purple line as a measure of labour progress: a longitudinal study. BMC Pregnancy Childbirth. 2010;10:54.

22. Enkin. M, Keirse. M, Neilson. J, Crowther. C, Duley. L, Hodnett. E. A guide to effective care in pregnancy and childbirth.

23. McKay S, Roberts J. Obstetrics by ear. Maternal and caregive perceptions of the meaning of maternal sounds during second stage labor. J Nurse Midwifery. 1990;35(5):266-73.

24. Hobbs L. Assessing cervical dilatation without VEs. Watching the purple line. Pract Midwife. 1998;1(11):34-5.

25. Byrne DL, Edmonds DK. Clinical method for evaluating progress in first stage of labour. Lancet. 1990;335(8681):122.

26. Pornprasertsuk W, Treetampinich C, Ayudhya N, O-Prasertsawa P, Rattanasiri S. Relationship between Alteration of Sacral Pain and Cervical Progression in Latent Phase of Labor : Diagnostic Study. Thai JObst Gynaecol. 2008;16:101-97. 